Fabs would inhibit the reassociation of subparticles, only one protein (L19) was inaccessible to antibodies in the $70 S$ particle itself. H. Roth (MaxPlanck-Institut, Berlin) has isolated more than twenty ribonucleoprotein fragments from $\mathrm{LiCl}$ cores of the $50 \mathrm{~S}$ subparticle by digestion with nuclease. The fragments, separated on sucrose gradients, contained from two to twenty-eight proteins. With a few notable exceptions, the data from these various sources were in good agreement, and two topographical models of the $30 \mathrm{~S}$ proteins were presented by $\mathrm{R}$. Traut and A. Bollen (Université de Bruxelles).

On the RNA front, C. Ehresmann (Institut de Biologie Moléculaire, Strasbourg) reported the latest improvements on the primary sequence of $16 \mathrm{~S}$ RNA, and C. Branlant (Institut de Biologic Moléculaire, Strasbourg) described some binding sites of $50 \mathrm{~S}$ proteins on the 23S RNA, in particular the complex formed between 5S RNA (with L5, L18 and L25) and 23S RNA in the presence of L2. As far as primary structure of individual proteins is concerned, it seems that L7 and L12 have been strongly conserved during evolution; two acidic proteins from rat liver were shown to be related immunologically to L7/L12 (G. Stöffler and I. Wool, Max-Planck-Institut, Berlin), and the corresponding proteins from the halophile Halobacterium cutirubrum showed large homologous sequences (A. Matheson, National Research Council of Canada, Ottawa). S5 has again turned up as an altered protein in several ribosomal mutants, notably in revertants from valyl-tRNA synthetase mutants described by $\mathrm{H}$. Wittman (Max-Planck-Institut, Berlin). K. Isono (Max-Planck - Institut, Berlin) also found alterations in the Bacillus stearothermophilus protein corresponding to S5 (as well as S12) in streptomycin-resistant mutants.

On the functional side, $\mathrm{S} 12$ was shown by A. Spirin (Academy of Sciences of the USSR, Poustchino) to exert a negative control over non-enzymatic translocation, since particles reconstituted without S12 showed increased activity in this assay. With the $50 \mathrm{~S}$ particle, $\mathrm{K}$. Nierhaus (Max-Planck-Institut, Berlin) found that L11 and L16 are the proteins responsible for chloramphenicol binding. L6 is also important for the chloramphenicol effect, but does not actually bind the antibiotic. L11 binds thiostrepton (J. Gordon and J. Highland, Friedrich Miescher Institut, Basel), and is also involved together with L.18 at the peptidyl transferase centre, as was shown by C. Cantor with a peptidyl tRNA photo-affinity label. E. Küchler (University of Vienna) also used a photo-affinity label to demonstrate that peptidyl tRNA can be bound to 23S RNA near to the binding site of the 5S RNA-protein complex. Evidence for conformational changes during protein synthesis came from $D$. Vasquez (Instituto de Biologia Celular, Madrid), who found that the antibiotic anisomycin binds to eukaryotic ribosomes with different affinities according to the functional state of the particles.

Looking at the more intimate details of tRNA binding, M. Sprinzl (MaxPlanck-Institut, Göttingen) used suitably modified tRNA analogues to demonstrate that tRNA synthetases attach the amino acids to the 2 -hydroxyl position of the terminal ribose. Elongation factor $T_{u}$ also recognises only a 2 '-acylated tRNA. Peptidyl transfer can be effected to either a 2'- or 3'acylated tRNA at the A site, but only from a $3^{\prime}$-acylated moiety at the P site. V. Erdmann (Max-Planck-Institut, Berlin) showed that the oligonucleotide $\mathrm{T} \psi \mathrm{CG}$ can be used instead of a complete de-acylated tRNA to stimulate magic spot synthesis.

Last but by no means least, $M$. Nomura (University of Wisconsin, Madison) reported some elegant experiments with $\lambda$ phages containing various amounts of the genetic material in the aroEstrA region of the $E$. coli genome. Using a DNA-dependent protein-synthesising system, he found that at least ten $30 \mathrm{~S}$ and fourteen $50 \mathrm{~S}$ proteins are encoded within this segment of DNA. The technique offers the possibility of detailed mapping of the ribosomal protein genes.

\section{How microorganisms damage plants}

from a Correspondent

IN plant, as in animal pathology much research effort has been directed towards investigating the mechanisms by which pathogens damage their hosts. One of the most important advances of recent years has been the realisation that in a small, although by no means unimportant group of plant pathogens, the ability to produce toxin is the major factor determining virulence towards specific hosts. The most convincing experiment to demonstrate this relationship was performed by Scheffer and his associates (Phytopathology, 57, 1288; 1967). They crossed two closely related toxin-producing fungi Cochliobolus victoriae and $C$. carbonum, pathogens respectively of oats and maize. $F_{1}$ offspring segregated in a 1: $1: 1: 1: 1$ ratio for the abilities to produce $C$. victoriae toxin, $C$. carbonum toxin, both toxins or no toxin. These progeny were respectively pathogenic on oats, maize, both oats and maize or neither host.

Attempts to elucidate the mechanism of action of these host-specific toxins have been stimulated by the knowledge that these toxins comprise some of the most potent biologically active substances known. Root growth of susceptible oat seedlings treated with $C$. victoriae toxin is prevented by as little as $0.0002 \mu \mathrm{g}$ toxin $\mathrm{ml}^{-1}$, a potency approaching that of the more active clostridial toxins. In susceptible plants almost any metabolic system that one chooses to examine is deranged by toxin treatment. Many of these changes are undoubtedly sccondary effects and evidence is accumulating that the primary site of action of at least some toxins resides in the plasmalemma. Supporting evidence for this hypothesis comes from the findings that toxin treatment of susceptible host cells leads to a rapid leakage of intracellular contents (Scheffer and Samaddar, Rec. Adv. Phytochem., 3, 123; 1970; Keck and Hodges, Phytopathology, 63, 226; $1973)$, to a rapid depolarisation of the plasmamembrane potential (Novacky and Hanchey, Physiol. Pl. Path., 4, $161 ; 1974)$ and also results in an inhibition of solute uptake and failure of the protoplasts to plasmolyse in hypertonic solutions.

Even more convincing evidence has come from the studies of Strobel and his associates ( $J$. biol. Chem., 248, 1321; 1973; Proc. natn. Acad. Sci., U.S.A., 70, 1693; 1973; ibid., 71, 1413; 1974) on the eyespot disease of sugar cane caused by Helminthosporium sacchari. The toxin produced by this fungus seems to be a primary determinant of disease and has been characterised as 2-hydroxy-cyclopropyl- $\alpha$-Dgalactopyranoside. Strobel has found that sensitivity to the toxin is correlated with the presence of a specific toxin-binding receptor protein in the plasmalemma of susceptible cells. Those cells resistant to the toxin possess an immunologically identical protein which does not bind the toxin. The specific proteins from resistant and susceptible cells have similar molecular weights, both consist of four subunits but differ slightly in electrophoretic mobility and with respect to four different amino acid residues.

This is the first positive identification of a specific chemical factor associated with disease susceptibility in a higher plant, an association long suspected on theoretical and genetical grounds. Although these reports establish the presence of a toxin-binding protein within the plasmalemma, much remains to be discovered as to the mechanism by which the toxin-protein interaction destroys the normal functioning of the membrane. These reports also illustrate another basic similarity between the functioning of animal and plant cells and suggest that the study of plant cell surfaces should prove useful. 